\title{
FOREWORD \\ OPTIMIZATION IN NETWORKS: MODELING, ALGORITHMS AND APPLICATIONS
}

\author{
Philippe Mahey ${ }^{1}$, Geraldo R. Mateus ${ }^{2}$, \\ Martín G. Ravetti ${ }^{2}$ and Maurício C. de Souza ${ }^{2}$
}

Network models provide insights to tackle a variety of problems in such diverse areas as production, logistics, routing, location, among others. Network structures are largely present in many industrial and service contexts like supply chain, transportation, communication and natural systems. Despite the fundamental developments achieved since the early days of Operations Research, the community still faces many challenges in the field, ranging from modeling paradigms for hard problems to the design and experimental evaluation of efficient methods and algorithms. Given its importance and applicability, this Special Issue of Pesquisa Operacional aims at presenting recent contributions inspired by real-life network optimization problems.

The result is this issue that features the following eight papers:

1 - Araujo et al. propose an alternative method to solve the multi-terminal maximum flow problem when the given network has cut nodes.

2 - Quilliot et al. propose a set of models and algorithms for current problems of vehicle sharing relocation.

3 - Ferone et al. provide an overview of the state-of-the-art and the current research trends concerning shortest paths problem on dynamic graphs.

4 - Shiina et al. propose a new approach based on a linear approximation to allocate loop controller devices in power delivery networks.

5 - Jaumard et al. tackle the problem of determining the minimum cost bandwidth assignment of the links in the network for which traffic.

6 - Benhamiche et al. investigate a variant of the multi-layer network design problem. The authors propose a formulation and a column generation procedure based on a Dantzig-Wolf decomposition.

\footnotetext{
${ }^{1}$ Guest editor: Université de Clermont Auvergne, France. E-mail: philippe.mahey @isima.fr

${ }^{2}$ Guest editors: Universidade Federal de Minas Gerais, Brazil.

E-mails: mateus@dcc.ufmg.br; Martin.ravetti@dep.ufmg.br; mauricio.souza@dep.ufmg.br
} 
7 - Mahey and Souza survey the models, and algorithmic issues of multicommodity flow networks with non-convex arcs cost functions.

8 - Kosuch, Letournel and Lisser study a stochastic version of the celebrated knapsack problem, which includes chance constraints and random item sizes.

We would like to thank the authors that submitted their papers to this special issue, the anonymous referees, and the editor of Pesquisa Operacional and the Brazilian Society of Operations Research that made the edition of this issue possible. 